\title{
Syndromes run together in the RAS pathway
}

istinct syndromes with partially overlapping phenotypes sometimes result from mutations in adjacent components of core signaling pathways shared by many cell types. Recent successes in identifying the causative mutations in a set of rare developmental syndromes highlight the importance of investigating phenotypic overlaps using the architecture of biochemical signaling networks. These findings are now assigning roles in human development to genes better known for their effects on dysregulation of cell signaling in cancer cells.

Ivan Le Lorraine Albright's 1929 painting "Among Those Left" depicts a blacksmith, a man of short stature whose widely spaced eyes angle downward under drooping eyelids toward his low, prominent ears. His shirt is open to reveal the hollow of his sternum. This may be the most famous portrait of someone with Noonan syndrome (Pediatrics 66, 468-469; 1980; reviewed in Am. J. Med. Genet. 66, 334-339; 1996). This autosomal dominant syndrome is common enough-at 1 in 1,000 to 1 in 2,500 live births - to be represented outside the specialist literature, unlike two extremely rare, sporadic conditions with overlapping features: cardio-facio-cutaneous (CFC) and Costello syndromes. Looking at this picture is puzzling because some of the man's features are individual or familial, some are imposed by the artist and some may be syndromic. Even from this superficial experience, a nonspecialist can readily appreciate some of the difficulties faced by the expert dysmorphologist in making a differential diagnosis among syndromes with overlapping characters.

About half of the individuals with Noonan syndrome have mutations in PTPN11, encoding a protein tyrosine phosphatase that functions in the ras pathway. Marco Tartaglia and colleagues (Nat. Genet. 29, 465-468; 2001) found mutations clustered at the interface between the phosphatase domain and amino-terminal SH2 domain within the protein. This presents an unusually large target for gain-of-function mutations, perhaps explaining (in addition to differences in severity) why Noonan syndrome is so much more prevalent than the other developmental syndromes of the RAS pathway.

Yoko Aoki and colleagues (Nat. Genet. 37, 1038-1040; 2005) found heterozygous de novo HRAS mutations in 12 of 13 individuals with Costello syndrome. These were mutations previously identified as somatic oncogenic mutations in tumors. The authors suggested that many of the phenotypes might reflect increased growth factor-induced cell proliferation and that hyperkeratosis of the skin, cardiac hypertrophy and macrocephaly might be due to altered cell proliferation, growth or cell death.

Now, on page 294, Tetsuya Niihori and colleagues identify two KRAS mutations and eight BRAF mutations in 19 out of 43 individuals with CFC. Rodriguez-Viciana et al. (Science, published online 26 January 2006; doi: 10.1126/science.1124642) found that 18 of 23 individuals with CFC had mutations in BRAF. In other individuals with CFC, they found mutations in MAP2K1 and MAP2K2, encoding downstream effectors of BRAF, but they found no mutations in HRAS or in PTPN11. Also in this issue, ( $\mathrm{p}$ 331) Suzanne Schubbert and colleagues report unprecedented de novo germline mutations in KRAS in individuals with Noonan and CFC syndromes and suggest that strong gain-of-function KRAS mutations may be incompatible with life.

In comparing the effort expended on understanding signaling pathways dysregulated in cancer with the insights gained from normal developmental roles, Weeber and Sweatt (Neuron $33,845-848$; 2002) recast the cAMP and ras pathways of the hippocampus as a 'learning pathway', considering the phenotypes of mutations in NF1 and CREBBP, located at either end of the signaling chain. They predicted that mutations in the genes encoding ras, raf, MEK and ERK would not be informative in understanding cognition, as they are likely to be lethal. Although Noonan syndrome is not usually associated with learning difficulties, NF1 mutations causing the overlapping NF1-Noonan syndrome (Am. J. Hum. Genet. 77, 1092-1101; 2005) are associated, in some cases, with mental retardation, and this phenotype is also found in the CFC and Costello syndromes.

Cardiovascular malformations are found more consistently across the syndromes of the RAS pathway, and it may be profitable to search for mutations in other pathway components by examining individuals with other syndromes that share cardiovascular phenotypes with the syndromes of the Noonan spectrum. Even here, it would be a mistake to restrict attention to the linear ras pathway, as PTPN11 regulates multiple signaling cascades. Uhlen et al. (Proc. Natl. Acad. Sci. USA 103, 2160-2165; 2006) recently found that gain-of-function PTPN11 mutations increased spontaneous $\mathrm{Ca}^{2+}$ oscillations in cardiac myocytes, thereby interfering with the transcriptional activity of NFAT. These observations suggest a mechanism whereby the PTPN11 mutations found in individuals with Noonan syndrome might interfere with cardiac morphogenesis. 